\title{
A Defective Angina Pectoris Pain Warning System: Experimental Findings of Ischemic and Electrical Pain Test ${ }^{1}$
}

\author{
Conrad Droste, Mark W. Greenlee * and Helmut Roskamm \\ Benedikt Kreutz Cardiovascular Rehabilitation Center, Bad Krozingen, and * Neurological Clinic, \\ Brain Research Unit, University of Freiburg, Freiburg (F.R.G.) \\ (Received 15 April 1985, revised received 9 December 1985, accepted 12 December 1985)
}

\begin{abstract}
Summary
Ischemic pain threshold and tolerance levels using the tourniquet pain technique and electrical cutaneous pain thresholds were measured in patients with asymptomatic ischemic heart disease. Thirty asymptomatic patients, who repeatedly exhibited no angina pectoris pain during the occurrence of exercise-induced coronary ischemia ( $\geqslant 0.1 \mathrm{mV}$ ST segment depression in exercise ECG) were compared to 30 randomly selected symptomatic control patients. In a smaller patient group (6 symptomatic, 6 asympatomatic) the degree of forearm ischemia during the tourniquet test was determined non-invasively by monitoring transcutaneous $\mathrm{pO}_{2}$. Results indicated that asymptomatic patients needed significantly more time to reach pain threshold following occlusion of forearm blood flow and exhibited significantly lower tcpO $\mathrm{O}_{2}$ values at threshold than symptomatic patients. Electrical pain thresholds were also elevated in the asymptomatic group. These findings indicate that the phenomenon of asymptomatic myocardial ischemia can be explained by an extracardiac pain modifying mechanism.
\end{abstract}

\section{Introduction}

Among patients with coronary heart disease, a small group has been found that is of interest to pain research. These patients have objective signs of severe ischemic

\footnotetext{
1 Part of these findings were presented at the IV World Congress on Pain, Seattle, WA, U.S.A., August, 1984.
} 
heart disease, but fail to experience angina pectoris pain. This phenomenon is referred to as asymptomatic myocardial ischemia. Although it has been known for some time that myocardial infarction can occur silently, more recently an increasing number of reports suggest that transient myocardial ischemia may also occur without pain $[4,6,15]$. Cohn [5] has described this as a defective anginal pain warning system that would normally signal the patient to stop activity. This lack of pain is of considerable importance for the prognoses of these patients, i.e., especially in cases of sudden cardiac death [10].

An explanation for this absence of pain in patients with asymptomatic myocardial ischemia remains equivocal. The aim of our study was to explore whether a general hyposensibility to pain may be responsible for this faulty warning system. We further asked whether endorphins play a role in the pain modification exhibited in these patients.

(1) By exercising the forearm after arterial occlusion, we measured the duration of work necessary to elicit an ischemic pain response (threshold) and that needed to reach tolerance levels in symptomatic and asymptomatic patients with proved myocardial ischemia.

(2) A possible explanation put forth for the lack of angina pectoris in asymptomatic myocardial ischemia suggests that these patients may not obtain the levels of ischemia during exercise that symptomatic patients do and that this lower ischemic level is insufficient to elicit angina pectoris pain. To test whether asymptomatic patients differ in their tissue oxygen metabolism, we measured the transcutaneous partial oxygen tension $[12,20]$ in the occluded forearm by employing a non-invasive polarographic oxygen sensor. This measure provides information concerning the oxygen supply and demand in the resting and working muscle. Following arterial occlusion, the development of ischemia and anoxia in the working forearm can thus be quantitatively assessed [see also 8].

(3) To test whether differences in pain thresholds were specific to ischemic pain, we measured thresholds for perceiving pain induced by electrical stimulation of the dermis following the method introduced by Notermans [22].

(4) To investigate the possible role of endogenous opioids (e.g., endorphins) in asymptomatic myocardial ischemia, we retested some of our asymptomatic patients on exercise ECG after injection of the opioid antagonist naloxone. The occurrence of pain during exercise following naloxone injection would suggest that such endogenous opioids might play a role in the absence of angina pectoris in patients with asymptomatic myocardial ischemia.

\section{Method}

\section{Ethical approval and informed consent}

The study was approved by the ethical board at the Rehabilitation Clinic in Bad Krozingen. At least 1 day before participating in the experiments, patients were given a written explanation of the general research aims and possible hazards 
involved and were then requested to sign an informed consent, which was complied to in all cases.

\section{Subjects}

Subjects were 60 male patients (mean age $51 \pm 7.2$ years) with severe ischemic heart disease.

(1) All patients demonstrated severe coronary artery disease $(\geqslant 75 \%$ stenosis in at least one major coronary vessel) as determined angiographically.

(2) All patients exhibited substantial ST segment depression ( $\geqslant 0.1 \mathrm{mV}$ in ECG) during several exercise conditions, thus representing an objective sign of transient myocardial ischemia.

(3) A total of 27 patients in the asymptomatic and 27 patients in the symptomatic group demonstrated a pathologically high pulmonary capillary pressure during exercise as measured by floating catheter, thus giving another objective sign of exercise-induced transient myocardial ischemia.

Patients were divided into one of two groups depending on the occurrence of self-reported pain during exercise ECG: 30 patients demonstrated symptomatic myocardial ischemia, i.e., the simultaneous occurrence of objective signs of myocardial ischemia and angina pectoris and 30 patients exhibited asymptomatic myocardial ischemia. These asymptomatic patients experienced no pain during the different exercise tests, although severe myocardial ischemica could be objectively shown. Of these 30 patients, 24 also reported no angina pectoris during everyday life, the remaining six had only mild symptoms, A total of 25 of these patients exhibited electrocardiographic evidence of previous myocardial infarction. In five of these patients, infarction occurred silently (i.e., no notice of infarction). Another seven of these patients noticed their infarction due to nausea or fatigue, but experienced no chest pain.

\section{Procedures}

In experiments 1 and 2 , ischemic pain thresholds and tolerance levels were recorded in patients by employing a modified version of the tourniquet technique, first introduced by Smith et al. [26]. Here, we requested our patients to engage with their non-preferred hand a hand-grip dynamometer at a rate of 40 beats $/ \mathrm{min}$. Exercise was performed until pain tolerance levels were reached. Ethical considerations led us to choose this form of the tourniquet technique, because the overall duration of the test was much shorter than with submaximal effort. Furthermore, the maximal effort technique was very similar to the exercise ECG tests performed clinically by the patients. In 4 symptomatic and in 3 asymptomatic, exercise was discontinued due to fatigue. In such cases, the cuff remained inflated and patients were asked to wait until tolerance was reached. Prior to engaging in this exercise, the cuff of a manometer was manually inflated to $170 \mathrm{~mm} \mathrm{Hg}$ (all patients had systolic blood pressure below $150 \mathrm{~mm} \mathrm{Hg}$ ), thereby completely occluding arterial flow to the forearm. The $\mathrm{tcpO}_{2}$ electrode used in experiment 2 further assured us that no leakage took place. After the cuff was fully inflated patients were asked to begin the hand-grip exercise. Patients were requested to report the following: 
(a) The time when pain was first perceived in the working forearm. We refer to this as the duration threshold.

(b) The time when the perceived pain became so intense that they could no longer tolerate it. We refer to this as the tolerance level.

In experiment 1 (30 symptomatic and 30 asymptomatic patients) the time elapsing between the start of stimulation to the (a) threshold and (b) tolerance levels was recorded.

In experiment 2 (in a randomly selected subgroup of 6 symptomatic and 6 asymptomatic patients) transcutaneous partial oxygen tension (tcpO $\mathrm{O}_{2}$ ) and skin blood flow (using heat clearance rate) were non-invasively monitored during the ischemic test using the Oxymonitor (Hellige Co.). This instrument consists of a polarographic sensor that is positioned on the skin surface of the forearm. The sensor has 3 polarographic cathodes that are submerged in electrolyte, all of which are covered with a Teflon membrane. A change in the amount of reduced oxygen in the electrolyte elicits a corresponding change in the potentials of these cathodes. The sensor is heated to $44^{\circ} \mathrm{C}$ thus eliciting hyperemia in the cutaneous tissue directly below the sensor. This provides a sufficient supply of oxygen in healthy cutaneous tissue so that in resting tcpO $\mathrm{O}_{2}$ approaches arterial $\mathrm{pO}_{2}$ [20]. Heart rate and respiration were simultaneously recorded. The time elapsing to threshold and tolerance levels was also assessed.

In experiment 3 , electrical pain thresholds were determined by the Notermans procedure [22] at 3 sites on the left thigh using 10 incremental and 10 decremental staircase measurements per site. The electrode was positioned immediately above the skin surface, upon which electrolyte paste had been applied. Electrical impulses were given for $5 \mathrm{msec}$ with an interstimulus interval of $5 \mathrm{sec}$. Patients responsed in a forced-choice paradigm whether they detected the impulse (i.e. pricking pain) or not. Skin resistance was simultaneously controlled. Average threshold values are based on the mean of 60 trials per subject. The Notermans procedure was preferred over other forms of electrical stimulation (i.e., tooth pulp stimulation), because, for our heart patients, we wanted a method that appeared the least hazardous.

In experiment 4,10 of our 30 asymptomatic patients were randomly selected to perform exercise ECG after intravenous injection of $2 \mathrm{mg}$ of the morphine antagonist naloxone. Injection was given immediately before the exercise test. As a placebo control, these patients were examined after injection of the same volume of saline. Naloxone and placebo injections were given in random order on a double-blind basis.

\section{Results}

All patients were administered a detailed neurologic examination including a tactile sensibility test using von Frey's hairs on different areas of the body surface. We did not find any differences on neurologic variables between these two groups. There was no evidence that would suggest polyneuropathy, e.g., such as history of higher alcohol consumption or influence of toxic substances, diabetic polyneuropa- 
TABLE I

COMPARISON OF SELECTED MEDICAL VARIABLES IN PATIENTS WITH SYMPTOMATIC. AND ASYMPTOMATIC MYOCARDIAL ISCHEMIA

ns = not significant.

\begin{tabular}{lccl}
\hline & \multicolumn{2}{l}{ Myocardial ischemia } & \\
\cline { 2 - 4 } & $\begin{array}{l}\text { Symptomatic } \\
(\mathrm{n}=30)\end{array}$ & $\begin{array}{l}\text { Asymptomatic } \\
(\mathrm{n}=30)\end{array}$ & $P$ \\
\hline 1-vessel disease & 10 \\
2-vessel disease & 7 \\
3-vessel disease & 13 & 4 \\
Friesinger score & $9.1 \pm 2.9$ & 16 & \\
Ejection fraction $(\%)$ & $58 \pm 14$ & $10.5 \pm 2.6$ & $\mathrm{~ns}$ \\
Heart volume (ml) & $814 \pm \mathbf{x}=2.4$ & $\mathrm{~ns}$ \\
Heart volume/body weight (ml/kg) & $10.8 \pm 1.2$ & $861 \pm 12$ & $\mathrm{~ns}$ \\
Previous myocardial infarction & 23 & $11.3 \pm 2.4$ & $\mathrm{~ns}$ \\
\hline
\end{tabular}

TABLE II

COMPARISON ON EXERCISE ECG VARIABLES BETWEEN SYMPTOMATIC $(n=30)$ AND ASYMPTOMATIC $(\mathrm{n}=30)$ PATIENT GROUPS

ns $=$ not significant.

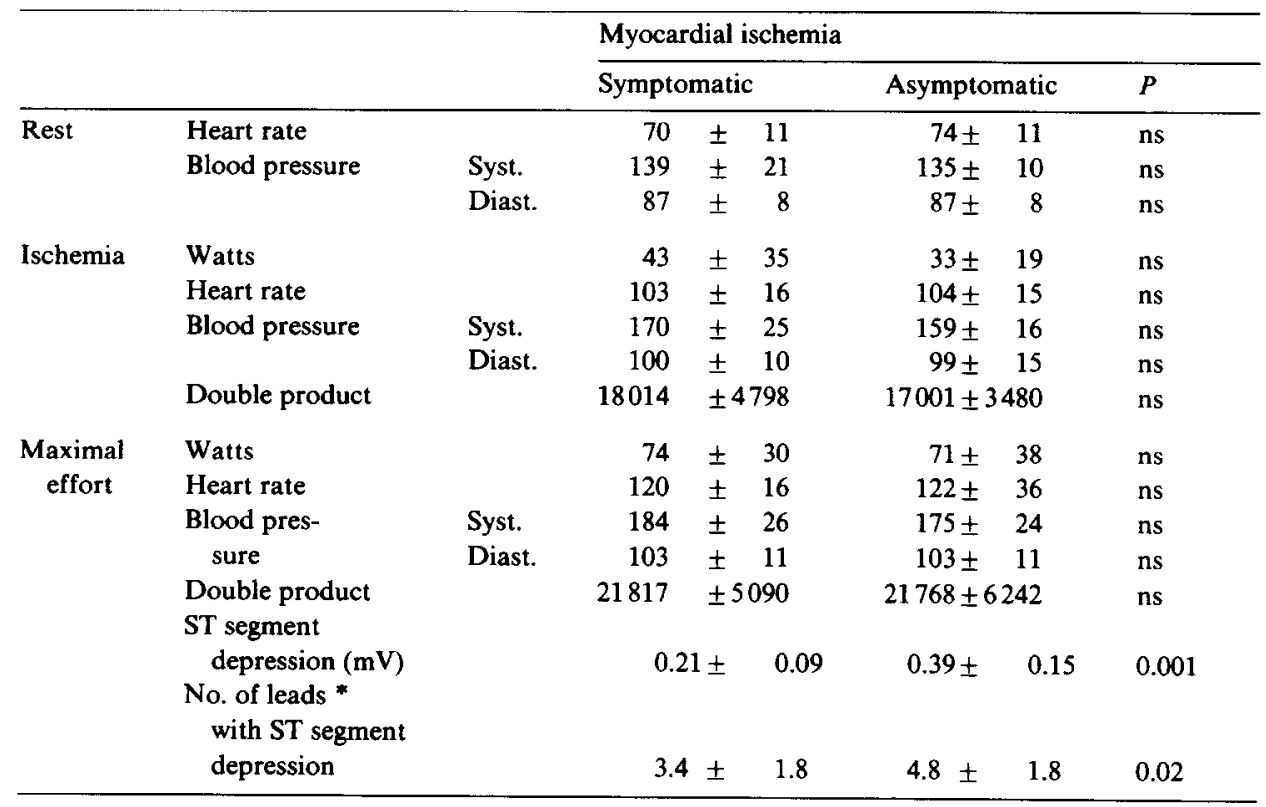

* Of a total of 12 leads. 
thy, or differences in tactile thresholds. No general innate insensibility was evident in these patients. All patients could experience pain and showed no signs of obvious neurologic deficits.

Table I presents the cardiologic data for these two patient groups. As is evident in the table, no significant differences were found between the patient groups on severity of coronary heart disease (1-, 2- and 3-vessel disease, the Friesinger score), ejection fraction, heart volume, heart volume related to body weight, and previous infarction. Also in standard coronary risk factors (i.e., age, smoking, hypertension, diabetes, cholesterol and triglyceride level), no significant difference was found between patient groups. In the symptomatic group, diabetes was present in 3 patients and in the asymptomatic group in 2 patients.

Table II shows the results of exercise ECG (supine bicycle ergometer). The only differences evident between our patient groups indicate that myocardial ischemia was more substantial in the asymptomatic group. ST segment depression was slightly more pronounced and occurred in more leads in the asymptomatic group. Extraneous factors that could have caused false positive ST segment depression (i.e., digitalis medication, hypopotassium levels, myocardial hypertrophy, valvular disease) or that could have influenced coronary ischemia and angina (medication, nitrates, beta-blockers, calcium antagonists) were controlled for.

\section{Experiment I}

The results of our first experiment are depicted in Fig. 1. Asymptomatic patients exhibited higher ischemic pain threshold and pain tolerance levels than did symptomatic patients; both values are statistically significant ( $t$ tests; $t=2.64, d f=58$, $P<0.01 ; t=1.75, d f=58, P<0.05$, one-tailed). For the symptomatic patients ischemic pain occurred after an average of $70.4 \mathrm{sec}$, while asymptomatic patients first reported pain after an average of $99.7 \mathrm{sec}$.

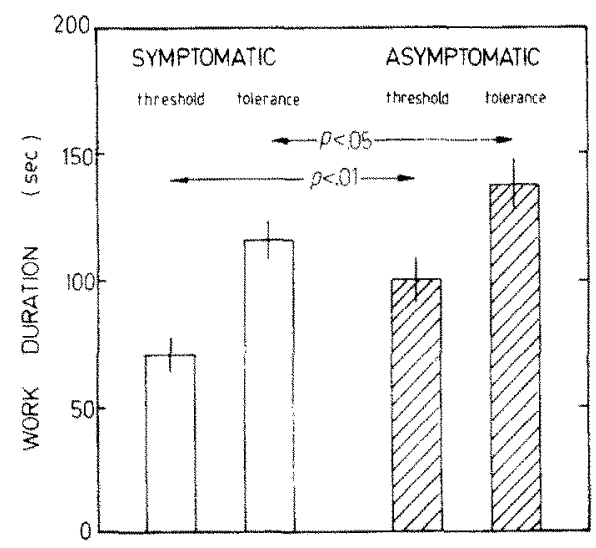

Fig. 1. Group differences on time needed to reach threshold and tolerance during the ischemic pain test. Results of $t$ tests shown for $(n=30)$ symptomatic and $(n=30)$ asymptomatic patients. Error bars show \pm 1 standard error of mean. 


\section{Experiment 2}

Fig. 2 presents the mean values for $\mathrm{cppO}_{2}$ (upper trace) and heart rate (lower trace) for symptomatic (dashed lines) and asymptomatic (solid lines) groups. As is evident in Fig. 2, the asymptomatic patients exhibited lower tcpO $\mathrm{O}_{2}$ levels at threshold and tolerance and needed more time to reach threshold and tolerance levels than do symptomatic patients.

Fig. 3 shows these differences in the form of histograms. On the left half of Fig. $3 \mathrm{~A}$, the mean values ( \pm 1 S.E.) for $t \mathrm{cpO}_{2}$ at threshold and tolerance levels are shown for the symptomatic (blank column) and asymptomatic (hatched column) groups. On the right side of Fig. 3B, the time elapsing from the start of the task to threshold and tolerance levels is also depicted for both patient groups. Though not depicted in Fig. 3, no significant differences were found for mean recovery time (defined as the time needed to regain the prestimulation baseline values for $t \mathrm{cpO}_{2}$ ) or for initial tcpO $\mathrm{O}_{2}$ baseline values, suggesting that normal blood flow and tissue $\mathrm{pO}_{2}$ levels were comparable between patient groups.

Significant differences ( $t$ tests, $t=2.25, d f=10, P<0.05$ ) were found for the time needed to reach threshold level and for the tcpO $\mathrm{O}_{2}$ values at threshold $(t=2.69$, $d f=10, P<0.05)$. Though substantial, the differences in tolerance levels $(t=2.02$, $d f=10, P<0.07$ and $t=2.09, d f=10, P<0.06$, for tcpO $\mathrm{O}_{2}$ and work duration, respectively) did not obtain customary significance levels. This is probably due to our small sample size $(n=12)$ for this experiment. The time needed to reach threshold for the asymptomatic group was greater than the time required by symptomatic patients to obtain tolerance levels (Fig. 3B). The corresponding tcpO values were lower at threshold in the asymptomatic group than at tolerance level for the symptomatic group (Fig. 3A).

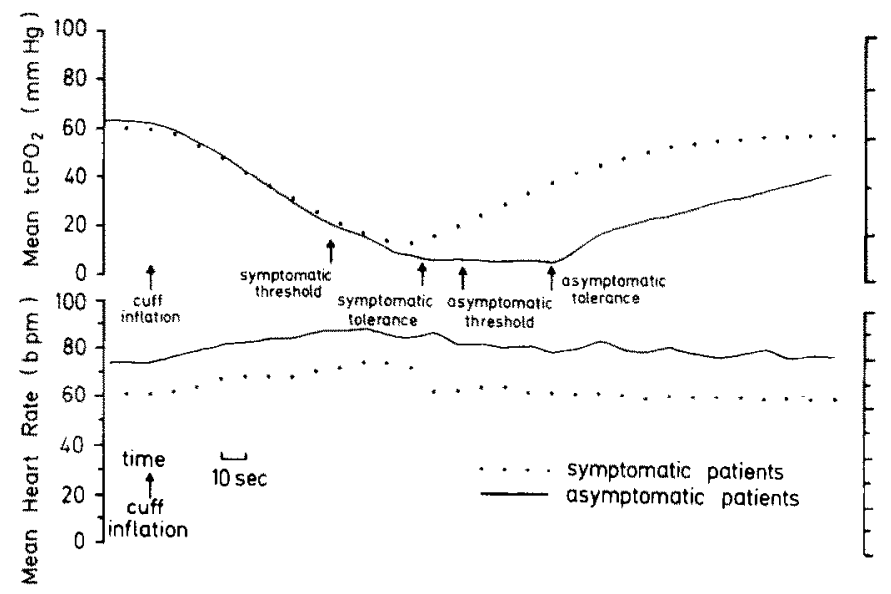

Fig. 2. Time course of mean values of tcpO $\mathrm{O}_{2}$ (upper traces) and heart rate (lower traces) for symptomatic (dashed lines; $n=6$ ) and asymptomatic (solid lines; $n=6$ ) patients. Time of cuff inflation denoted by arrows. Threshold and tolerance levels are depicted separately (arrows) for symptomatic and asymptomatic groups. 

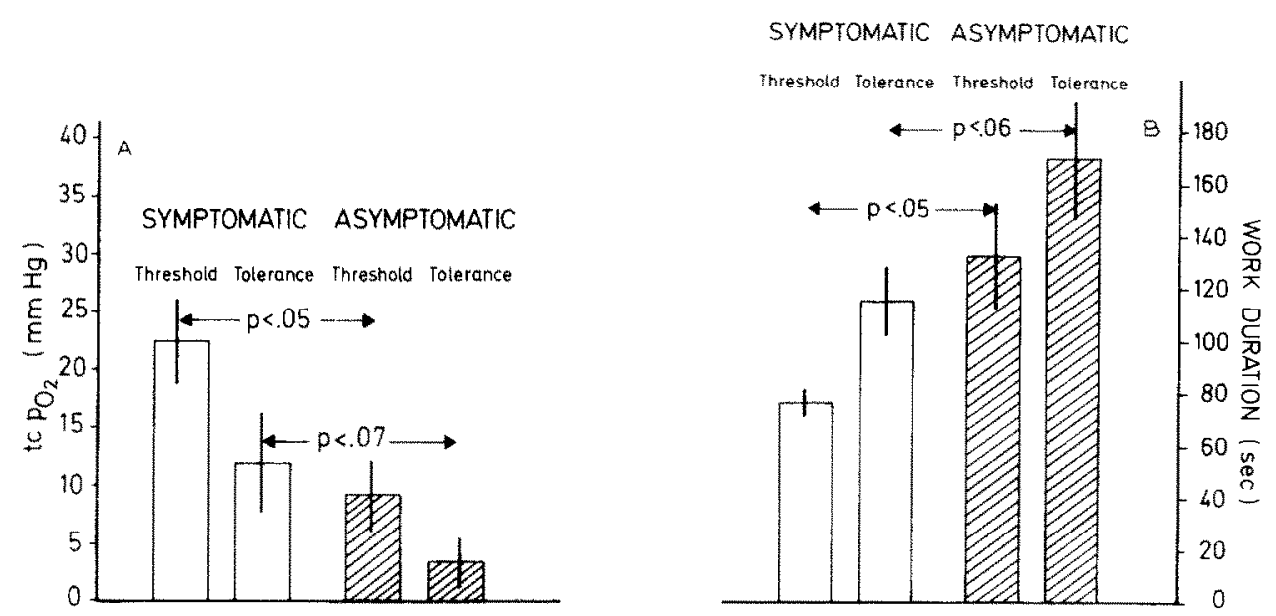

Fig. 3. A: group differences in tcpO $\mathrm{O}_{2}$ at threshold and tolerance levels. Mean values are depicted for $(n=6)$ symptomatic patients by blank columns and those for $(n=6)$ asymptomatic patients by hatched columns. Error bars show \pm 1 S.E. Significance levels are shown for $t$ tests. B: group differences in time needed to reach threshold and tolerance levels for symptomatic $(n=6)$ and asymptomatic $(n=6)$ patients. Significance levels are shown for $t$ tests.

\section{Experiment 3}

Significant differences in threshold were exhibited between the symptomatic and asymptomatic groups on the electrical pain test. Results are depicted in Fig. 4. As can be seen in the figure, symptomatic patients showed a mean threshold of 0.55 $\mathrm{mA}$, which is in good agreement with Noterman's original findings [22]. In contrast, asymptomatic patients demonstrated a significantly higher mean threshold of 1.0 $\mathrm{mA}$. Results of a $t$ test on group differences for the mean threshold value were

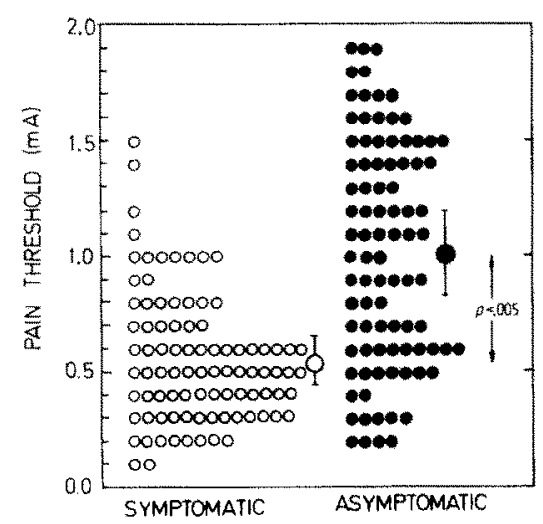

Fig. 4. Mean values \pm 1 S.E. and distribution of individual values on the electrical pain test according to the Notermans procedure for symptomatic $(O)$ and asymptomatic (e) groups. Significance levels are shown for $t$ tests. 
highly significant $(t=7.81, d f=58, P<0.005)$. Skin resistance had no significant effect on threshold levels.

\section{Experiment 4}

Following naloxone injection, no differences were evident in parameters of exercise ECG, neither in maximum effort, maximum heart rate, maximum blood pressure, nor in ST segment depression in asymptomatic patients. Only in 2 of our 10 asymptomatic patients could angina pectoris be provoked after naloxone injection, although they had never previously experienced such symptoms during exercise testing. In 1 patient the effect of naloxone was dose dependent. After $2 \mathrm{mg}$ of naloxone, angina pectoris occurred at $75 \mathrm{~W}, 2 \mathrm{~min}$ and increased at $75 \mathrm{~W}, 4 \mathrm{~min}$. After $4 \mathrm{mg}$ of naloxone, the patient reported typical angina pectoris at $50 \mathrm{~W}$ and at $75 \mathrm{~W}$ the exercise test was discontinued due to severe angina. These findings were replicated after a 2 month period. This patient had a 1-vessel disease without infarction and was totally asymptomatic. The other asymptomatic patient in whom angina pectoris could be provoked during exercise ECG had a 2-vessel disease and an inferior infarction. Although the other asymptomatic patient did report pain during exercise after naloxone injection, his pain did not increase by continuing exercise.

\section{Discussion}

The findings of the experiments presented here indicate that significant and replicable differences in threshold and tolerance levels for experimentally induced pain exist between symptomatic and asymptomatic myocardial ischemia patients, asymptomatic patients showing a general hyposensitivity to nociceptive stimulation. Earlier clinical reports of patients with painless myocardial infarction [1,24] suggest that these patients have an unusually low sensibility to pain. However, these earlier findings were only partly quantified [19]. Keele [17] and Procacci et al. [23] reported higher pain thresholds in patients with silent myocardial infarction. The observation that, in most of our asymptomatic patients, asymptomatic ischemia was reproducible over a number of examinations and was evident in daily life and during infarction itself speaks for a more person-related rather than a situation-dependent cause. Note, however, that there is considerable within-group variance (Figs. 1 and 4). It appears, therefore, that other factors may be involved in asymptomatic myocardial ischemia.

It has been argued that destruction of pain pathways following myocardial infarction, diffuse coronary heart disease, or polyneuropathy may be responsible for asymptomatic myocardial ischemia. This argument appears, however, not likely to apply to our patients. Symptomatic and asymptomatic patients showed no differences on history of prior infarction, number and degree of diseased coronary vessels, history of alcohol consumption or diabetes.

Other authors $[11,14,25]$ have argued that patients with asymptomatic myocardial ischemia may exhibit lower tissue oxygen demands and thus do not obtain the levels 
of ischemia during exercise that symptomatic patients do. This argument does not hold for our patients because: (1) ST segment depression was greater in our asymptomatic patients (as seen in Table II) and (2) no mean difference was found between the rate of residual oxygen metabolism (as indicated by the rate of decrease in $\mathrm{tcpO}_{2}$ ) in the working forearm following arterial occlusion. This lack of differences is evident in the initial tcpO $\mathrm{O}_{2}$ values and the mean slope (representing $\mathrm{O}_{2}$ decrement rate) to the point of cuff deflation (see Fig. 2). As is evident in that figure, asymptomatics worked longer thus creating greater levels of anoxia before pain was perceived.

Our results rather suggest that cardiac pain is influenced by an extracardiac, pain-regulating factor, which may inhibit nociceptive pathways either at the dorsal root or higher up in the central nervous system. The observation that in some asymptomatic patients angina pectoris could be provoked following naloxone, but not following placebo, support a possible role of opioid mechanisms.

Janal et al. [16] showed that endogenous opioid mechanisms are specifically involved in the modification of deep somatic pain, similar to ischemic pain. Moore et al. [21] and Lewis et al. [18] found substantial interindividual differences in sensitivity to ischemic pain. In addition, Yanagida [28] and Willer et al. [27] showed that congenital insensitivity to other forms of clinical pain in single cases can be reversed by medium-to-large doses of naloxone. The results of Buchsbaum and coworkers $[2,3]$ suggest that varying concentrations of endorphins in plasma and cerebrospinal fluid or varying amounts of endorphins in intersynaptic fluid may represent a chemical substrate of individual differences in pain sensitivity.

Most of the asymptomatic patients tested here reported no pain following injection of $2 \mathrm{mg}$ naloxone. Although substantial, angina pectoris could be provoked in only 2 of 10 asymptomatic patients tested in exercise ECG following naloxone injection. These single observations are, therefore, not sufficient to support a relationship between myocardial ischemia and endorphins. Han [13] has, however, recently argued that not all opioid-dependent analgesic mechanisms can be influenced by low-to-moderate doses of naloxone as those used here. Other factors may be involved in this diminished sensitivity to pain in patients with asymptomatic myocardial ischemia, for example, non-opioid pain regulating mechanisms. Further research should also consider the role of cognitive processes that define the presence or absence of ischemic pain [9]. Basic biochemical (e.g., arachidonic acid metabolites) and mechanical factors involved in coronary pain [7,8], also remain unclear.

\section{Acknowledgements}

M.W.G. was supported by the Deutsche Forschungsgemeinschaft (SFB 70, A6). Many thanks go to $\mathrm{C}$. Wollert and $\mathrm{H}$. Leist (Hellige, Freiburg) for their helpful technical advice and for use of the Oxymonitor in experiment 2. 


\section{References}

1 Babey, A.M., Painless acute information of the heart, New Engl. J. Med., 220 (1939) 410-412.

2 Buchsbaum, M.S., Davis, G.C., Coppola, R. and Naber, D., Opiate pharmacology and individual differences. I. Psychophysical pain measurements, Pain, 10 (1981) 357-366.

3 Buchsbaum, M.S., Davis, G.C., Cuppula, R. and Naber, D., Opiate pharmacology and individual differences. II. Somatosensory evoked potentials, Pain, 10 (1981) 367-377.

4 Cohn, P.F., Severe asymptomatic coronary artery disease: a diagnostic, prognostic and therapeutic puzzle, Amer. J. Med., 62 (1977) 565-568.

5 Cohn, P.F., Silent myocardial ischemia in patients with a defective anginal warning system, Amer. J. Cardiol., 45 (1980) 697-702.

6 Cohn, P.F., Asymptomatic conorary artery disease, Mod. Concepts cardiovasc. Dis., 50 (1981) 55-60.

7 Droste, C., Neurophysiologie des Koronarschmerzes. In: H. Roskamm (Ed.), Handbuch der inneren Medizin, Vol. IX/3: Koronarerkrankungen, Springer, Berlin, 1984, pp. 157-174.

8 Droste, C. and Greenlee, M.W., Letter to the Editor. Two separate components of pain produced by the submaximal effort tourniquet technique, Pain, 23 (1985) 95-96.

9 Droste, C. und Roskamm H., Kognitive Einflüsse auf Angina Pectoris Schmerz im Belastungs-EKG, Z. Kardiol., 72, Suppl. (1983) 74.

10 Feruglio, G.A., Sudden death in patients with asymptomatic coronary heart disease. In: W. Rutishauser and H. Roskamm (Eds.), Silent Myocardial Ischemia, Springer, Berlin, 1984, pp. 144-150.

11 Gettes, L.S., Painless myocardial ischemia, Chest, 66 (1974) 612-613.

12 Greenlee, M.W. and Akita, M., Transcutaneous partial oxygen tension and skin blood flow monitoring: noninvasive measures on cardiorespiratory change, Biol. Psychol., 20 (1985) 285-294.

13 Han, J.S., Letter to the Editor, Pain, 21 (1985) 307-308.

14 Holmgreen, A., Hemodynamic sequences in asymptomatic exercise-induced ischemia. In: W. Rutishauser and H. Roskamm (Eds.), Silent Myocardial Ischemia, Springer, Berlin, 1984, pp. 66-70.

15 Iskandrian, A.S., Segal, B.L. and Anderson, G.S., Asymptomatic myocardial ischemia, Arch. intern. Med., 141 (1981) 95-97.

16 Janal, M.N., Colt, E.W.D., Clark, W.C. and Glusman, M., Pain sensitivity, mood and plasma endocrine levels in man following long-distance running: effects of naloxone, Pain, 19 (1984) 13-25.

17 Keele, K.D., A physician looks at pain. In: M. Weisenberg (Ed.), Pain. Clinical and Experimental Perspectives, Mosby, Saint Louis, MO, 1975, pp. 45-52.

18 Lewis, T., Pickering, G.W. and Rothschild, P., Observations upon muscular pain in intermittent claudication, Heart (Lond.), 15 (1931) 360-383.

19 Libman, E., Observations on individual sensitiveness to pain, J. Amer. med. Ass., 102 (1934) 335-341.

20 Lübbers, D.W., Theoretical basis of transcutaneous blood gas measurement, Crit. Care Med., 9 (1981) 721-733.

21 Moore, P.A., Duncan, G.H., Scott, D.S., Gregg, J.M. and Ghia, J.M., The submaximum effort tourniquet test: its use in evaluating experimental and chronic pain, Pain, 6 (1979) 382-395.

22 Notermans, S.L.H., Measurements of the pain threshold determined by electrical stimulation and its clinical application. In: M. Weisenberg (Ed.), Pain. Clinical and Experimental Respectives, Mosby, Saint Louis, MO, 1975, pp. 72-87.

23 Procacci, P., Zoppi, M., Padeletti, L. and Maresca, M., Myocardial infarction without pain. A study of sensory function of the upper limbs, Pain, 2 (1976) 309-313.

24 Rosemann, M.D., Painless myocardial infarction: a review of the literature and analysis of 220 cases, Ann. intern. Med., 41 (1954) 1-8.

25 Sigwart, U., Grbic, M., Payot, M., Goy, J.J., Essinger, A. and Fisher, A., Ischemic events during coronary artery balloon obstruction. In: W. Rutishauser and H. Roskamm (Eds.), Silent Myocardial Ischemia, Springer, Berlin, 1984, pp. 29-36.

26 Smith, G.M., Lowenstein, E., Hubbard, J.H. and Beecher, H.K., Experimental pain produced by the submaximum effort tourniquet technique: further evidence of validity, J. Pharmacol. exp. Ther., 163 (1968) 468-474.

27 Willer, J.C., Dehen, H., Bourneau, F. and Cambier, J., Congenital insensitivity to pain and naloxone, Lancet, ii (1978) 739.

28 Yanagida, H., Congenital insensitivity and naloxone, Lancet, ii (1978) 520. 